\title{
AN INEQUALITY FOR THE EIGENVALUES OF A CLASS OF SELF-ADJOINT OPERATORS
}

\author{
BY WILLIAM STENGER ${ }^{1}$
}

\section{Communicated by J. B. Diaz, January 9, 1967}

1. Introduction. The theory of intermediate problems introduced by $\mathrm{A}$. Weinstein in 1935 has not only been of great importance in its application to the numerical computation of lower bounds for eigenvalues, but has also been useful in the investigation of many general questions of a purely theoretical nature. Among these results, let us mention Weinstein's new maximum-minimum theory [1], [2], Stenger's analogous treatment of Poincaré's inequalities [3], and a remarkable inequality of Aronszajn [5, p. 476]. In this paper we give a seemingly new inequality, which is in a sense complementary to Aronszajn's inequality.

2. The new inequality. Let $A$ be a self-adjoint linear operator, defined on a dense subspace $D$ of a complex Hilbert space $H$ having the scalar product $(u, v)$. Let $H^{\prime}$ be a closed subspace of $H$, and let $H^{\prime \prime}=H \ominus H^{\prime}$. Denote by $P^{\prime}$ and $P^{\prime \prime}$ the projections on to $H^{\prime}$ and $H^{\prime \prime}$, respectively. We assume that the lower parts of the spectra of (i) $A$, (ii) $P^{\prime} A P^{\prime}$, and (iii) $P^{\prime \prime} A P^{\prime \prime}$ consist of isolated eigenvalues $\lambda_{1} \leqq \lambda_{2}$ $\leqq \cdots, \lambda_{1}^{\prime} \leqq \lambda_{2}^{\prime} \leqq \cdots$, and $\lambda_{1}^{\prime \prime} \leqq \lambda_{2}^{\prime \prime} \leqq \cdots$, each having finite multiplicity. Obviously, these hypotheses on (i), (ii) and (iii) are satisfied for a nonpositive self-adjoint compact $A$. Moreover, many important Schroedinger-type operators satisfy the hypothesis for (i). It has been recently shown [4] that, under certain conditions, the hypotheses are also true for (ii) and (iii). Let $u_{1}, u_{2}, \cdots, u_{1}^{\prime}, u_{2}^{\prime}, \cdots$, and $u_{1}^{\prime \prime}, u_{2}^{\prime \prime}, \ldots$ be the corresponding orthonormal sequences of eigenvectors.

THEOREM. The eigenvalues of the operators $A, P^{\prime} A P^{\prime}$, and $P^{\prime \prime} A P^{\prime \prime}$ satisfy the inequality

$$
\lambda_{1}+\lambda_{i+j} \leqq \lambda_{i}^{\prime}+\lambda_{j}^{\prime \prime}, \quad i, j=1,2, \cdots .
$$

Proof. Let $R(u)$ denote the Rayleigh quotient $(A u, u) /(u, u)$. For any $u \in H,(u, u)=1$, we can write $u=\sigma v+\tau w$, where $v \in H^{\prime}, w \in H^{\prime \prime}$, $(v, v)=(w, w)=1$, and $|\sigma|^{2}+|\tau|^{2}=1$. If $\tau=0$, we have $u=v, R(u)$ $=R(v)$, and $\lambda_{1} \leqq R(w)$ for any $w$. Therefore, we have

$$
R(u)+\lambda_{1} \leqq R(v)+R(w) .
$$

${ }^{1}$ Research partially sponsored by the Air Force Office of Scientific Research, Office of Aerospace Research, United States Air Force under AFOSR Grant 400-64. 
We prove now that (2) is true for $\tau \neq 0$. In fact, we have by direct computation

$$
\begin{aligned}
0 \leqq \frac{R(u)-\lambda_{1}}{|\tau|^{2}}= & \left|\frac{\sigma}{\tau}\right|^{2}\left[R(v)-\lambda_{1}\right]+\left(\frac{\sigma}{\tau}\right)\left(\left[A-\lambda_{1} I\right] v, w\right) \\
& +\left(\frac{\tilde{\sigma}}{\tau}\right) \overline{\left(\left[A-\lambda_{1} I\right] v, w\right)}+\left[R(w)-\lambda_{1}\right] .
\end{aligned}
$$

Now we apply the following lemma, which was used in the proof of Aronszajn's inequality, see [6, p. 76].

Lemma. Let $\alpha, \gamma$ be any nonnegative constants and let $\beta$ be any complex number such that

$$
f(z)=\alpha z \bar{z}+\beta z+\overline{\beta z}+\gamma \geqq 0
$$

for all complex $z$. Then we have

$$
f(z) \leqq(1+z \bar{z})(\alpha+\gamma) .
$$

Putting $z=\sigma / \tau, \alpha=R(v)-\lambda_{1}, \beta=\left(\left[A-\lambda_{1} I\right] v, w\right)$ and $\gamma=R(w)-\lambda_{1}$, we apply the inequality (4) in (3) and obtain again the inequality (2). Therefore (2) is true for every $u \in H$. Now let $U_{i}^{\prime}$ $=\operatorname{sp}\left\{u_{1}^{\prime}, u_{2}^{\prime}, \cdots, u_{i}^{\prime}\right\}$ and $U_{j}^{\prime \prime}=\operatorname{sp}\left\{u_{1}^{\prime \prime}, u_{2}^{\prime \prime}, \cdots, u_{j}^{\prime \prime}\right\}$ for $i, j=1,2, \cdots$ Using the inequality (2) and a minimum-maximum principle, see [3], we get

$\lambda_{i+j} \leqq \max _{u \in U_{i}^{\prime} \oplus U_{j}^{\prime \prime}} R(u) \leqq \max _{v \in U_{i}^{\prime}} R(v)+\max _{w \in U_{i}^{\prime \prime}} R(w)-\lambda_{1}=\lambda_{i}^{\prime}+\lambda_{j}^{\prime \prime}-\lambda_{1}$ which proves the theorem.

The inequality (1) is a counterpart to the inequality of Aronszajn

$$
\lambda_{i}^{\prime}+\lambda_{j}^{\prime \prime} \leqq \lambda_{i+j-1}, \quad i, j=1,2, \cdots,
$$

which is true for nonpositive compact operators. In another paper we shall give the necessary and sufficient conditions, on the spaces $H^{\prime}$ and $H^{\prime \prime}$, in order that equality hold in the inequality (5).

3. Application. We now give an application of our inequality (1) to the uniform estimation of the error in Weinstein's lower bounds for eigenvalues. Let $p \in D$ such that $(p, p)=1$ and $(A p, p) \neq 0$, and let $H^{\prime}=\operatorname{sp}\{p\}$. Then $\lambda_{1}^{\prime}=R(p)$ is the only nonzero eigenvalue of $P^{\prime} A P^{\prime}$ and is a Poincaré-Rayleigh-Ritz upper bound for $\lambda_{1}$. The numbers $\lambda_{1}^{\prime \prime} \leqq \lambda_{2}^{\prime \prime} \leqq \cdots$ are the eigenvalues of the first intermediate problem 


$$
A u-(A u, p) p=\lambda u, \quad(u, p)=0,
$$

and satisfy the inequality

$$
\lambda_{j}^{\prime \prime} \leqq \lambda_{1+j}, \quad j=1,2, \cdots .
$$

Applying our inequality (1), we have

$$
0 \leqq \lambda_{1+j}-\lambda_{j}^{\prime \prime} \leqq \lambda_{1}^{\prime}-\lambda_{1}, \quad j=1,2, \cdots .
$$

This inequality shows that the intermediate problem associated with a given vector $p$ yields lower bounds for the higher eigenvalues which are at least as accurate as the Poincaré-Rayleigh-Ritz upper bound for $\lambda_{1}$ obtained with the same $p$.

4. Remark. Let us point out here that Aronszajn's inequality (5) should not be confused with the following essentially different inequality due to Weyl $[10$, p. 445$]$.

THEOREM. Let $K, K^{(1)}$ and $K^{(2)}$ be compact self-adjoint operators on $H$ such that $K=K^{(1)}+K^{(2)}$. Then the nonpositive eigenvalues $\lambda_{1} \leqq \lambda_{2}$ $\leqq \cdots, \lambda_{1}^{(1)} \leqq \lambda_{2}^{(1)} \leqq \cdots$, and $\lambda_{1}^{(2)} \leqq \lambda_{2}^{(2)} \leqq \cdots$ of $K, K^{(1)}$, and $K^{(2)}$ satisfy the inequality

$$
\lambda_{i}^{(1)}+\lambda_{j}^{(2)} \leqq \lambda_{i+j-1}, \quad i, j=1,2, \cdots .
$$

This inequality comes directly from a maximum-minimum principle, while Aronszajn's inequality (5) requires the above lemma, in addition to a maximum-minimum principle.

For recent expositions of the theory of intermediate problems see the books by G. Fichera [7] and S. H. Gould [8] and the paper of J. B. Diaz [9].

\section{REFERENCES}

1. A. Weinstein, Intermediate problems and the maximum-minimum theory of eigenvalues, J. Math. Mech. 12 (1963), 235-246.

2. - An invariant formulation of the maximum-minimum theory of eigenvalues, J. Math. Mech. 16 (1966), 213-218.

3. W. Stenger, On Poincarés bounds for higher eigenvalues, Bull. Amer. Math. Soc. 72 (1966), 715-718.

4. - The maximum-minimum principle for the eigenvalues of unbounded operators, Notices Amer. Math. Soc. 13 (1966), 731.

5. N. Aronszajn, The Rayleigh-Ritz and A. Weinstein methods for approximation of eigenvalues, Proc. Nat. Acad. Sci. U.S.A. 34 (1948), 474-480.

6. H. Hamburger and M. E. Grimshaw, Linear transformations in n-dimensional vector space, Cambridge Univ. Press, Cambridge, 1951.

7. G. Fichera, Linear elliptic differential systems and eigenvalue problems, SpringerVerlag, New York, 1965. 
8. S. H. Gould, Variational methods for eigenvalue problems. An introduction to the Weinstein method of intermediate problems, 2nd ed., Univ. of Toronto Press, Toronto, 1966.

9. J. B. Diaz, Upper and lower bounds for eigenvalues, Proceedings of the Eighth Symposium on Applied Mathematics (American Mathematical Society), McGrawHill, New York, 1958.

10. H. Weyl, Das asymptotische Verteilungsgesetz der Eigenwerte linearer partieller Differentialgleichungen, Math. Ann. 71 (1911), 441-469.

Institute for Fluid Dynamics and Applied Mathematics, UNIVERSITY OF MARYLAND 